\title{
STREET REGION DETECTION FROM NORMALIZED DIGITAL SURFACE MODEL AND LASER DATA INTENSITY IMAGE
}

\author{
T. S. G. Mendes ${ }^{a} *$, A. P. Dal Poz ${ }^{b}$ \\ UNESP, São Paulo State University, Brazil \\ ${ }^{\text {a }}$ Cartographic Sciences Graduate Program - tatisussel@gmail.com \\ ${ }^{\mathrm{b}}$ Department of Cartography - aluir@fct.unesp.br
}

KEY WORDS: Street region detection, laser scanner data, normalized Digital Surface Model, intensity image, image processing techniques

\begin{abstract}
:
The urban road network extraction process can be simplified by firstly detecting regions corresponding to streets, allowing a substantial reduction of the search area. As a result, the extraction process is benefited in two aspects: the computational complexity and the reliability. This paper aims at detecting street regions using only data obtained by Laser Scanner Systems. A sequence of standard image processing techniques is used to process height and intensity laser scanner data. A normalized Digital Surface Model is derived from height laser scanner data, from which regions corresponding to aboveground objects (mainly trees and buildings) are detected. Next, detected tree regions are eliminated from the aboveground regions, remaining only buildings. Then, morphological operators are applied in order to obtain elongated street ribbons and homogeneous block regions. Street regions are also detected in the intensity image. The results obtained from the radiometric and geometric laser scanner data are combined, allowing the elimination of non-street regions and the improvement of the geometry of region boundaries. The experimental results showed that the methodology proved to be efficient to detect street regions.
\end{abstract}

\section{INTRODUCTION}

Automated urban road network extraction from digital images is an extremely complex task, since in urban environments the scenes involve a lot of objects that interact with streets. This problem can be simplified by detecting previously regions corresponding to street (RoI - Region of Interest), resulting in a considerable reducing of the search area. As a result, the computational effort and the reliability of the extraction process are improved. The use of only aerial or orbital images in the extraction process can suffer some limitations, mainly related to the similarity of the spectral response between the street and the other objects (e.g. buildings with gray roof). In order to overcome this problem, other data sources can provide additional information to support the extraction process and improve the results, as can be found in Hinz \& Baumgartner (2003) that integrated high-resolution images with a DSM (Digital Surface Model) and in Zhang (2004) that integrated colour images with pre-existing spatial data base.

The use of the information from the laser scanner data can contribute as additional information. Related works can be found in $\mathrm{Hu}$ et al. (2004) and Zhu et al. (2004), which integrated aerial image and laser data and also in Tiwari et al. (2009) that integrated IKONOS images and altimetry laser data.

Some methodologies for road network extraction used only information obtained from laser scanner. Alharthy \& Bethel (2003) used laser pulse intensity to detected candidate pixels corresponding to road and height data to removed noise. In Clode et al. (2004) laser points are classified into points of road and non-road. The classification is based on a height range where the roads can be found and a range of laser pulse intensity according to the type of material of the roads.

\footnotetext{
* Corresponding author.
}

Streets in laser scanner data present peculiar characteristics. In normalized Digital Surface Model (NDSM), generated from 3D point cloud, the streets have homogeneous height values and are free of the occlusions caused by shadows of objects. In intensity image they appear in dark gray value, due to the low reflectance (approximately 17\%) in relation of the laser pulse (Wehr \& Lohr, 1999). On the one hand, the streets can be easily identified but, on the other hand, several objects (e.g. vegetation) present similar responses.

This paper presents a sequence of image processing techniques applied in both laser scanner data - intensity and NDSM images - whose goal is the detection of street regions. An important aspect of this work is the exploration of complementary characteristics of streets in these data sources.

\section{METHODOLOGY}

The flowchart of the proposed methodology is present in Figure 1. A 3D point cloud and intensity images, both obtained by Laser Scanner Systems, are used as input data. From the point cloud data is generated a NDSM image, which is the basis for detecting elongated street regions and homogeneous block regions. Candidate street regions are detected using the intensity image. The results are combined for the detection of the street regions. 


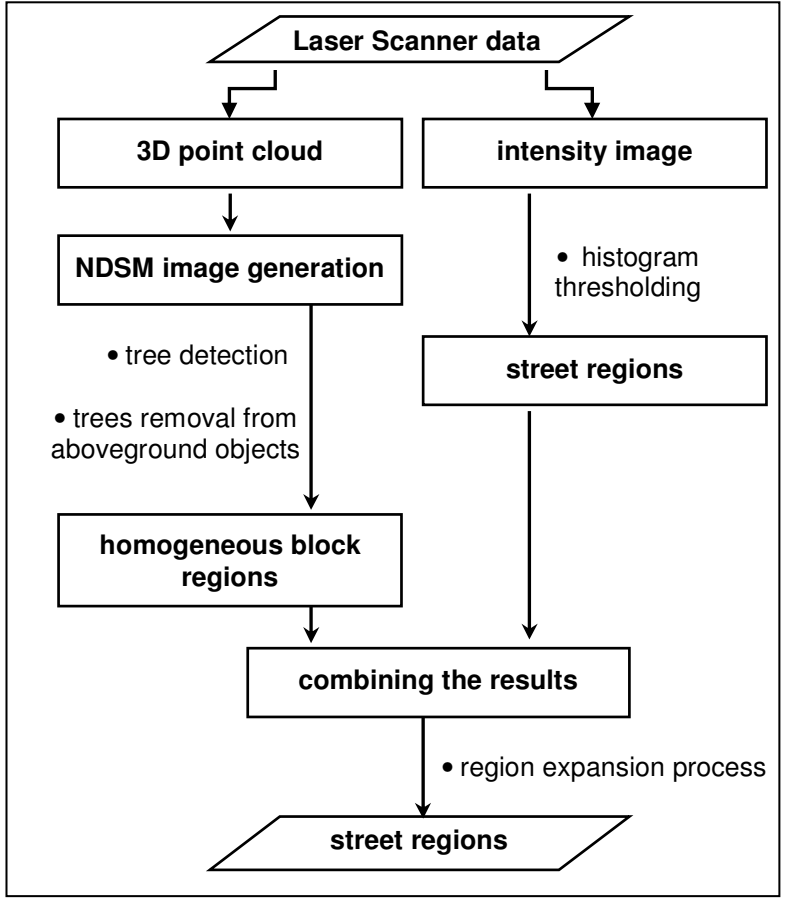

Figure 1. Proposed methodology flowchart.

\subsection{Material}

The study area is an urban area of Curitiba, Brazil. It is a residential area characterized by buildings, paved streets and vegetation of different sizes. The laser scanner data for this region were obtained using Optech ALTM System.

The experiments were performed using software HALCON 7.1 developed by MVTec (Machine Vision Technology).

\subsection{NDSM Image Generation}

Initially, the point cloud data is interpolated to generate a regular grid (DSM) using the nearest neighbour interpolator with resolution of 0.3 meters. Points representing the ground surface are collected in the DSM and another grid is generated, i. e. the DTM (Digital Terrain Model). For a representation of aboveground objects on a flat surface, a NDSM is obtained by subtracting the DTM from the corresponding DSM. The Figure 2 shows de image representing the NDSM.

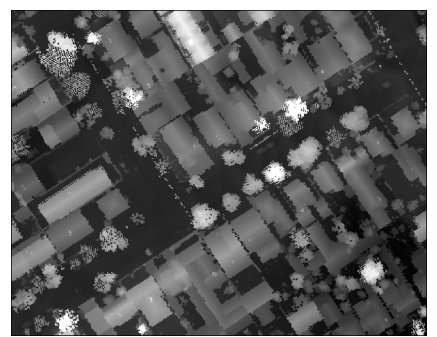

Figure 2. Image representing the NDSM.

\subsection{Trees Detection from the NDSM Image}

Regions corresponding to trees are detected based on texture and shape that these objects present in the NDSM image. For trees detection are necessary some steps, which are described in the following subsections.
2.3.1 Texture Filter: Trees are heterogeneous regions in NMDS image and present a texture that differentiates them from buildings (see Figure 2). Using a standard-deviation texture filter, heterogeneous regions are enhanced. This filter calculates the standard deviation of gray levels within a rectangular mask, whose dimensions (height and width) are parameters that must be provided. This operation results in a standard deviationvalued image, which is presented in Figure 3.

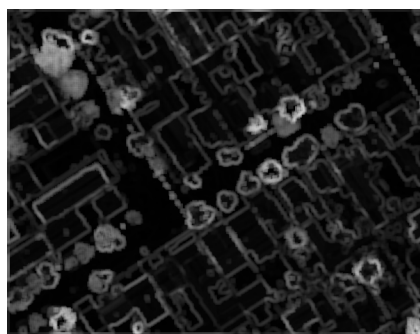

Figure 3. Standard deviation image derived from the NDSM.

2.3.2 Histogram Thresholding: In a standard deviation image (Fig. 3), the edges and trees are enhanced. Through the histogram analysis of this image is possible to choose a threshold value and to isolate such regions applying a histogram thresholding. However, not only trees but also some enhanced building contours are detected, as can be seen in Figure 4.

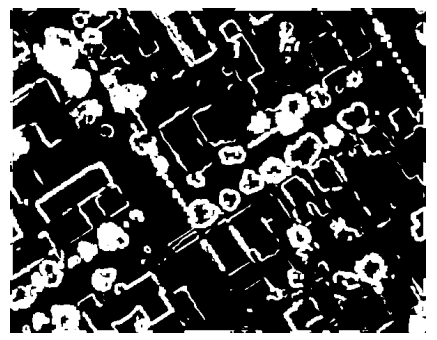

Figure 4. Regions enhanced detected from standard deviation image.

2.3.3 Morphological Operators: To eliminate non-tree regions obtained in previous step, it is assumed that trees usually have circular shape, while other objects have different shapes. Thus, morphological operators of opening and closing are applied using a disk shaped structuring element. The opening operator removes isthmus and islands, if these regions were smaller than structuring element. The closing operator fills gulfs and holes of regions smaller than structuring element. Figure 5 shows the contour of the tree regions obtained overlaid on the NDSM image.

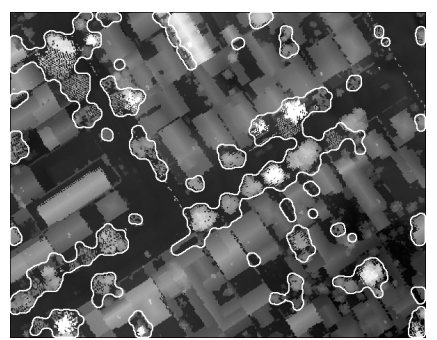

Figure 5. Contours of tree regions detected. 


\subsection{Trees Removal from Aboveground Objects}

In a NDSM image, the street have gray level values near zero, while the aboveground objects have gray level values according to their true heights, as can be seen in Figure 2. Based on these characteristics it is possible to separate the aboveground objects through a histogram thresholding, resulting in the binary image, where the aboveground objects are represented in white, while the ground is represented in black, as showed in Figure 6.

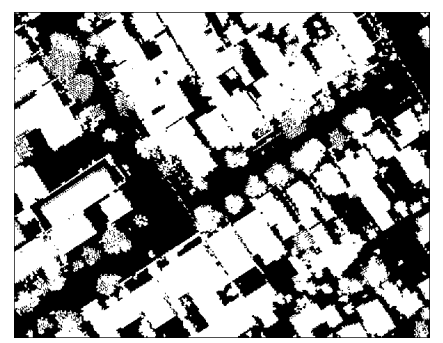

Figure 6. Binary image representing the aboveground objects.

Trees removal from aboveground objects is performed by a difference operation between the aboveground regions previously obtained (Fig. 6) and the trees detected (Section 2.3). Thus, only regions corresponding to buildings remain. Consequently, the regions that represent blocks become irregular and with holes inside (Fig. 7).

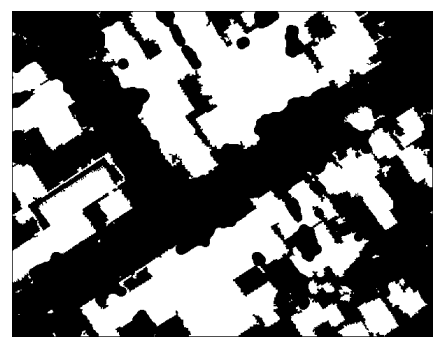

Figure 7. Trees removed from aboveground objects.

\subsection{Homogeneous Block Regions}

In order to obtain blocks as homogeneous regions and then obtain elongated and homogeneous street ribbons, morphological operators are applied in the previous result. The operators of dilation and erosion, both using a disk shaped structuring element, generate more regular regions, since the dilation operator fills regions smaller than the structuring element and the erosion operator clear regions smaller than the structuring element. Figure 8 shows streets as elongated ribbons, without occlusions caused by trees, and blocks as homogeneous regions.

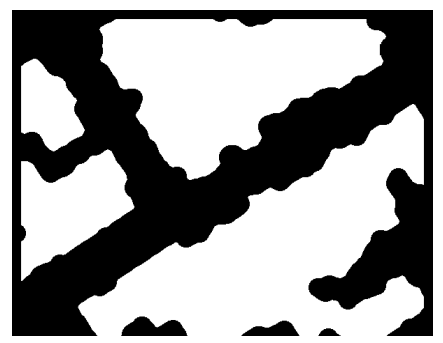

Figure 8. Elongated street ribbons and homogeneous blocks.

\subsection{Street Detection from Intensity Image}

Taking advantage of the street characteristics in the intensity image, it is possible to detect candidate regions corresponding to streets applying a histogram thresholding in the intensity image. The result is a binary image representing the street regions in white and the background objects in black. Figure 9 shows the intensity image (Fig. 9(a)) and the resulting binary image (Fig. 9(b)).

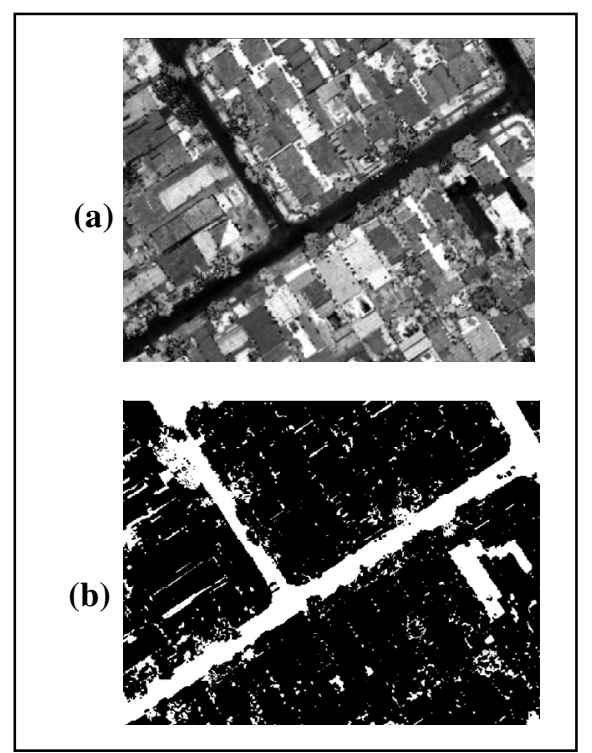

Figure 9. (a) Intensity image (b) Street regions detected by histogram thresholding.

The intensity image presents some objects that have radiometric responses similar to streets, and thus they are also detected in the histogram thresholding process.

\subsection{Street Region Detection Combining the Results Obtained from Intensity and NDSM Images}

The homogeneous regions representing the blocks obtained from the NDSM (Section 2.5) are combined with the candidate street regions obtained from an intensity image (Section 2.6). For this, block regions are expanded iteratively until they touch the street regions, called the forbidden areas. The expanding region operator works by adding or removing a pixel strip to a region. In this process, the non-street regions that appear inside the blocks (see Fig.9) are removed. Application of this process is shown in Figure 10, which represents the blocks, street and background regions in white, grey, and black, respectively. Figure 10(a) shows the regions before the expanding process. Expanded block regions are presented in Figure 10(b). 
(a)

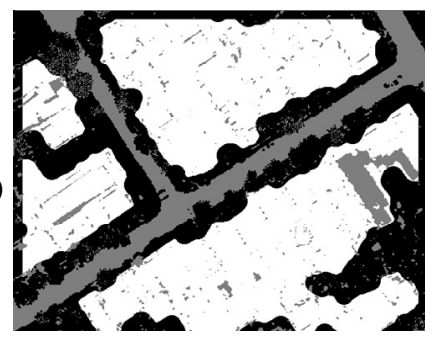

(b)

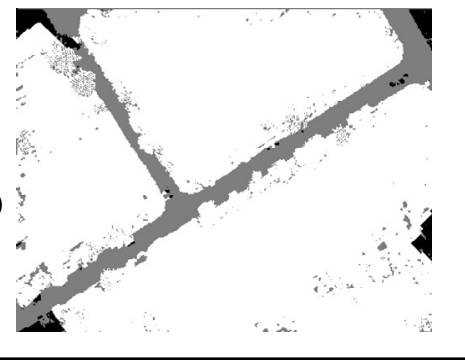

Figure10. (a) Block regions before the expanding region process. (b) Expanded block regions.

In order to obtain more regular block region boundaries, morphological operators of opening and closing are applied. We used a disk shaped structuring element. An example of result is shown in Figure 11(a). Figure 11(b) presents obtained block regions boundaries overlaid on the intensity image. Please, observe that boundary is very near to true block boundary.

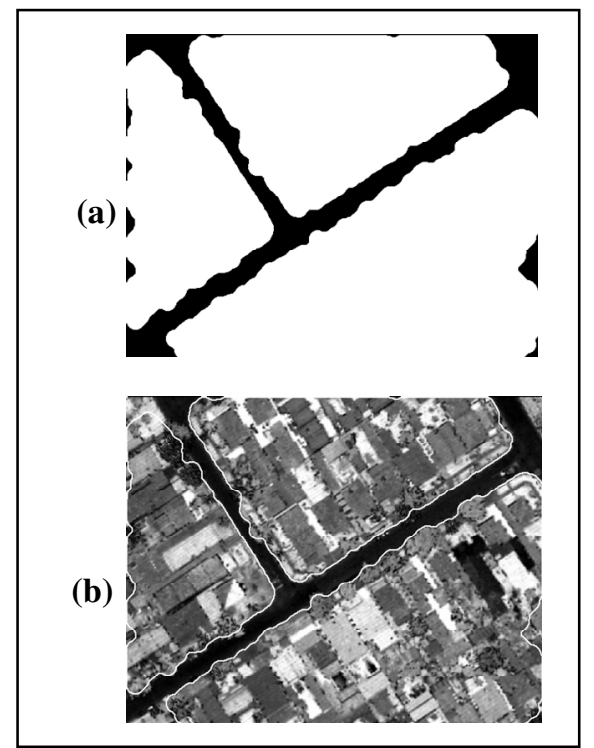

Figure 11. (a) Block regions after application of the morphological operators. (b) Block regions boundary overlaid on the intensity image.

The street region can be obtained as the complement of block regions detected previously, as shown in Figure 12. Note that street regions are successfully detected.

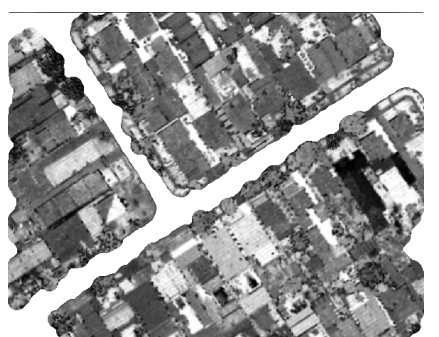

Figure 12. Result of the methodology for detect street regions overlaid on the intensity image.

\section{RESULTS}

In addition to results presented along with the methodology, more experiments were performed using another patch of the available laser data (see Figures 13, 14 e 15).

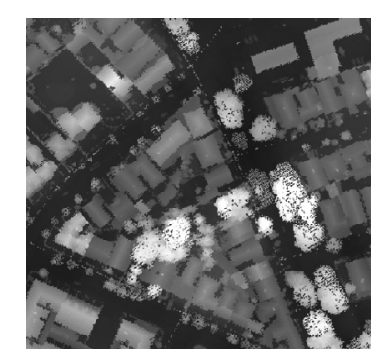

(a)

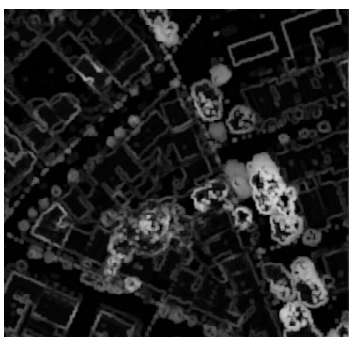

(c)

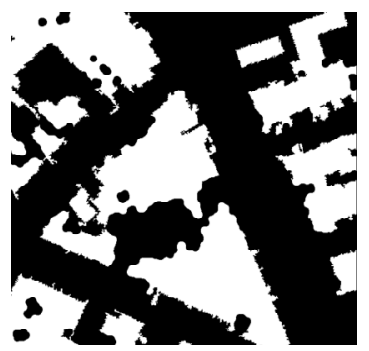

(e)

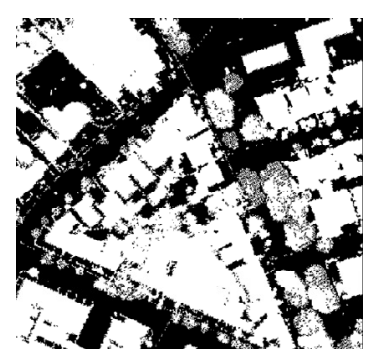

(b)

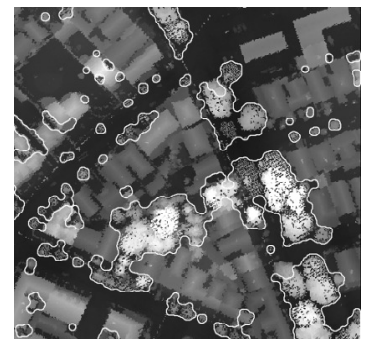

(d)

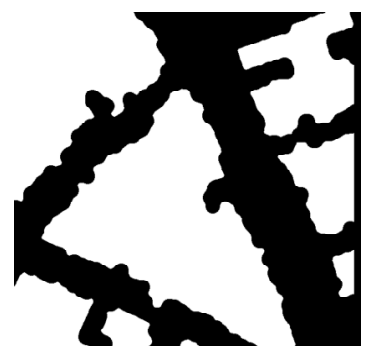

(f)
Figure 13. Results obtained from the NDSM image. (a) NDSM image. (b) Binary image representing the aboveground objects.

(c) Standard deviation image derived from the NDSM. (d)

Detected tree contours overlaid on the NDSM image. (e) Trees removed from aboveground objects. (f) Elongated street ribbons and homogeneous block regions.

Figure 13 presents the results obtained using the NDSM image. Figure 13(a) shows the NDSM image generated from the point 
cloud data. Note that nearly the right boundary of the image there are several trees occluding almost completely the street. The aboveground objects detected by histogram thresholding are shown in Figure 13(b). Figure 13(c) shows the standarddeviation image derived from NDSM, with enhanced trees along the streets and inside the blocks. The building edges also are enhanced. Figure 13(d) presents the contours of trees detected, showing that trees are successfully detected. The difference between aboveground objects and tree regions detected is shown in Figure 13(e). The streets (black elongated ribbons) and blocks (white homogeneous regions) are presented in Figure 13(f), which also shows that the holes inside de blocks are filled.

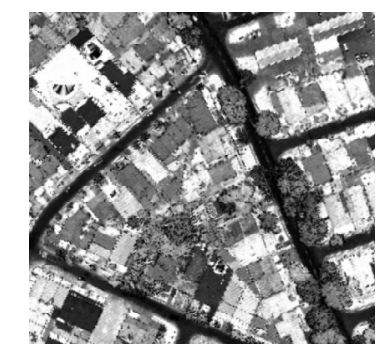

(a)

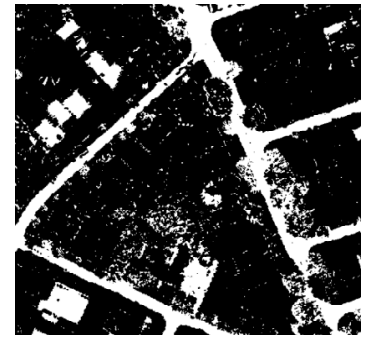

(b)
Figure 14. Results obtained from the intensity image. (a) Intensity image. (b) Binary image representing the street regions.

Figure 14(a) shows the results obtained using the intensity image. The result of the histogram thresholding is shown in Figure 14(b). Note several non-street regions, which are detected due to their similar radiometric response to our object of interest (streets).

Figure 15(a) presents the block regions (in white) obtained from the NDSM image and elongated regions (in gray) obtained through the application of the histogram thresholding to the intensity image. The elimination of the non-street regions is carried out by applying the expanding region process to these regions. Figure 15(c) shows the blocks region boundaries regularized by the application of the morphological operators. Detected street regions are overlaid on the intensity image (see Figure 15(d)). Again, the methodology proved to be efficient in detecting streets occluded by several trees.

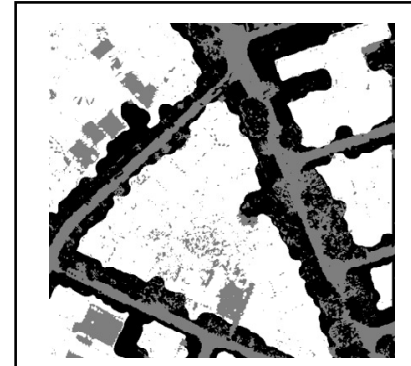

(a)

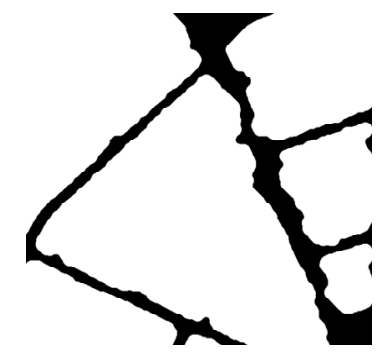

(c)

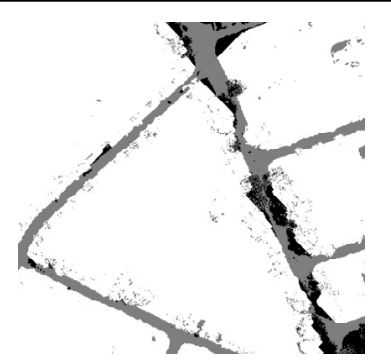

(b)

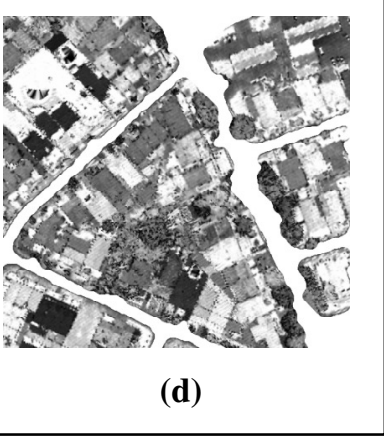

Figure 15. Results obtained combining the results generated from the radiometric and geometric laser data. (a) Block regions before the application of the region expansion process. (b)

Expanded block regions. (c) Block regions after application of the morphological operators. (d) Detected street regions overlaid on the intensity image.

\section{CONCLUSION}

This paper presented a methodology for street region detection by using the following laser scanner data: the NDSM image generated from height data and the intensity image. The steps of the methodology were described and two experiments were presented. From a visual analysis of the obtained results it is possible to conclude that street regions were successfully detected.

Refinements and improvements are planned, mainly to regularize street region contours detected by the proposed methodology.

\section{REFERENCES}

Alharthy, A. \& Bethel, J. 2003. Automated Road Extraction from LIDAR Data. In: Proceedings of ASPRS. Anchorage.

Clode, S., Kootsookos, P. \& Rottensteiner, F. 2004. The automatic extraction of roads from LIDAR data. In: The International Archives of the Photogrammetry, Remote Sensing and Spatial Information Science. Istanbul, Vol. XXXV, pp. 231-237.

Hinz, S. \& Baumgartner, A. 2003. Automatic extraction of urban road networks from multi-view aerial imagery. ISPRS Journal of Photogrammetry and Remote Sensing, 58(1-2), pp. 83-98. 
Hu, X., Tao, C. V. \& Hu, Y. 2004. Automatic road extraction from dense urban area by integrated processing of high resolution imagery and LIDAR data. In: The International Archives of the Photogrammetry, Remote Sensing and Spatial Information Sciences. Istanbul, Vol. XXXV, pp. 320-325.

Tiwari, P. S., Pande, H. \& Pandey, A. K. 2009. Automatic urban road extraction using Airborne Laser Scanning/altimetry and high resolution satellite data. Journal of the Indian Society of Remote Sensing, 37(2), pp. 223-231.

Wehr, A. \& Lohr, U. 1999. Airborne LASERscanning - an introduction and overview. ISPRS Journal of Photogrammetry and Remote Sensing. 54 (2-3), pp. 68-82.

Zhang, C. 2004. Towards an operational system for automated updating of road databases by integration of imagery and geodata. ISPRS Journal of Photogrammetry and Remote Sensing, 58, pp. 166-186.

Zhu, P., Lu, Z., Chen, X., Honda, K. \& Eiumnoh, A. 2004. Extraction of city roads through shadow path reconstruction using LASER data. Photogrammetric Engineering and Remote Sensing. 70 (12), pp. 1433-1440.

\section{ACKNOWLEDGEMENTS}

This paper is part of a $\mathrm{Ph}$. D. research supported by the Brazilian National Agency for Science and Technology (CNPq). The authors thank the LACTEC (Curitiba-Pr, Brazil) for providing the data for this research. 